\title{
Improved and Simplified Inapproximability for $k$-means
}

\author{
Euiwoong Lee ${ }^{\mathrm{a}, 1}$, Melanie Schmidt ${ }^{\mathrm{a}, 2}$, John Wright ${ }^{\mathrm{a}, 3}$ \\ ${ }^{a}$ Computer Science Department \\ Carnegie Mellon University \\ Pittsburgh, PA 15213
}

\begin{abstract}
The $k$-means problem consists of finding $k$ centers in $\mathbb{R}^{d}$ that minimize the sum of the squared distances of all points in an input set $P$ from $\mathbb{R}^{d}$ to their closest respective center. Awasthi et. al. recently showed that there exists a constant $\varepsilon^{\prime}>0$ such that it is NP-hard to approximate the $k$-means objective within a factor of $1+\varepsilon^{\prime}$. We establish that $1+\varepsilon^{\prime}$ is at least 1.0013 .
\end{abstract}

For a given set of points $P \subset \mathbb{R}^{d}$, the $k$-means problem consists of finding a partition of $P$ into $k$ clusters $\left(C_{1}, \ldots, C_{k}\right)$ with corresponding centers $\left(c_{1}, \ldots, c_{k}\right)$ that minimize the sum of the squared distances of all points in $P$ to their corresponding center, i.e. the quantity

$$
\arg \min _{\left(C_{1}, \ldots, C_{k}\right),\left(c_{1}, \ldots, c_{k}\right)} \sum_{i=1}^{k} \sum_{x \in C_{i}}\left\|x-c_{i}\right\|^{2}
$$

where $\|\cdot\|$ denotes the Euclidean distance. The $k$-means problem has been well-known since the fifties, when Lloyd [10] developed the famous local search heuristic also known as the $k$-means algorithm. Various exact, approximate, and heuristic algorithms have been developed since then. For a constant number of clusters $k$ and a constant dimension $d$, the problem can be solved by enumerating weighted Voronoi diagrams [7]. If the dimension is arbitrary but the number of centers is constant, many polynomial-time approximation schemes are known. For example, [6] gives an algorithm with running time $\mathcal{O}\left(n d+2^{\text {poly }(1 / \varepsilon, k)}\right)$. In the general case, only constant-factor approximation algorithms are known [8, 9], but no algorithm with an approximation ratio smaller than 9 has yet been found.

Surprisingly, no hardness iesults for the $k$-means problem were known even as recently as ten years ago. Today, it is known that the $k$-means problem is NP-hard, even for constant $k$ and arbitrary dimension $d[1,4]$ and also for arbitrary $k$ and constant $d[12]$. Early this year, Awasthi et. al. [2] showed that

\footnotetext{
${ }^{1}$ Supported by the Samsung Scholarship and NSF CCF-1115525.

${ }^{2}$ Supported by the German Academic Exchange Service (DAAD).

${ }^{3}$ Supported by a Simons Fellowsinip in Theoretical Computer Science.
}

Preprint submitted to Elsevier

September 2, 2015 
there exists a constant $\varepsilon^{\prime}>0$ such that it is NP-hard to approximate the $k$ means objective within a factor of $1+\varepsilon^{\prime}$. They use a reduction from the Vertex Cover problem on triangle-free graphs. Here, one is given a graph $G=(V, E)$ that does not contain a triangle, and the goal is to compute a minimal set of vertices $S$ which covers all the edges, meaning that for any $\left(v_{i}, v_{j}\right) \in E$, it holds that $v_{i} \in S$ or $v_{j} \in S$. To decide if $k$ vertices suffice to cover a given $G$, they construct a $k$-means instance in the following way. Let $b_{i}=(0, \ldots, 1, \ldots, 0)$ be the $i$ th vector in the standard basis of $\mathbb{R}^{|V|}$. For an edge $e=\left(v_{i}, v_{j}\right) \in E$, set $x_{e}=b_{i}+b_{j}$. The instance consists of the parameter $k$ and the point set $\left\{x_{e} \mid e \in E\right\}$. Note that the number of points is $|E|$ and their dimension is $|V|$.

A relatively simple analysis shows that this reduction is approximationpreserving. A vertex cover $S \subseteq V$ of size $k$ corresponds to a solution for $k$ means where we have centers at $\left\{b_{i}: v_{i} \in S\right\}$ and each point $x_{\left(v_{i}, v_{j}\right)}$ is assigned to a center in $S \cap\left\{b_{i}, b_{j}\right\}$ (which is nonempty because $S$ is a vertex cover). In addition, it can also be shown that a good solution for $k$-means reveals a small vertex cover of $G$ when $G$ is triangle-free.

Unfortunately, this reduction transforms $(1+\varepsilon)$-hardness for Vertex Cover on triangle-free graphs to $\left(1+\varepsilon^{\prime}\right)$-hardness for $k$-means where $\varepsilon^{\prime}=O\left(\frac{\varepsilon}{\Delta}\right)$ and $\Delta$ is the maximum degree of $G$. Awasthi et. al. 2] proved hardness of Vertex Cover on triangle-free graphs via a reduction from general Vertex Cover, where the best hardness result of Dinur and Safra 5 , has an unspecified large constant $\Delta$. Furthermore, the reduction uses a sophisticated spectral analysis to bound the size of the minimum vertex cover of a suitably chosen graph product.

Our result is based on the observation that hardness results for Vertex Cover on small-degree graphs lead to hardness of Vertex Cover on triangle-free graphs with the same degree in an extremely simple way. Combined with the result of Chlebík and Chlebíková [3] that proves hardness of approximating Vertex Cover on 4-regular graphs within $\approx 1.02$, this observation gives hardness of Vertex Cover on triangle-free, degree- 4 graphs without relying on the spectral analysis. The same reduction from Vertex Cover on triangle-free graphs to $k$ -

means then proves APX-hardness of $k$-means, with an improved ratio due to the small degree of $G$.

\section{Main Result}

Our main result is the following theorem.

Theorem 1. It is NP-hard to approximate $k$-means within a factor 1.0013.

We prove hardness of $k$-means by a reduction from Vertex Cover on 4regular graphs, for which we have the following hardness result of Chlebík and Chlebíková [3].

Theorem 2 ([3], see also Appendix A). Given a 4-regular graph $G=(V(G), E(G))$, it is NP-hard to distinguish to distinguish the following cases.

- $G$ has a vertex cover with at most $\alpha_{\min }|V(G)|$ vertices. 
- Every vertex cover of $G$ has at least $\alpha_{\max }|V(G)|$ vertices.

Here, $\alpha_{\min }=\left(2 \mu_{4, k}+8\right) /\left(4 \mu_{4, k}+12\right)$ and $\alpha_{\max }=\left(2 \mu_{4, k}+9\right) /\left(4 \mu_{4, k}+12\right)$ with $\mu_{4, k} \leq 21.7$. In particular, it is $N P$-hard to approximate Vertex Cover on degree-4 graphs within a factor of $\left(\alpha_{\max } / \alpha_{\min }\right) \geq 1.0192$.

Given a 4-regular graph $G=(V(G), E(G))$ for Vertex Cover with $n:=$ $|V(G)|$ vertices and $2 n$ edges, we first partition $E(G)$ into $E_{1}$ and $E_{2}$ such that $\left|E_{1}\right|=\left|E_{2}\right|=|E(G)| / 2=n$ and such that the subgraph $\left(V(G), E_{2}\right)$ is bipartite. Such a partition always exists: every graph has a cut containing at least half of the edges (well-known; see, e. g., [13]). Choose $n$ of these cut edges for $E_{2}$ and let $E_{1}$ be the remaining edges. We define $G^{\prime}=\left(V\left(G^{\prime}\right), E\left(G^{\prime}\right)\right)$ by splitting each edge in $E_{1}$ into three edges. Formally, $G^{\prime}$ is given by

$$
\begin{aligned}
& V\left(G^{\prime}\right)=V(G) \cup\left(\bigcup_{e=(u, v) \in E_{1}}\left\{v_{e, u}^{\prime}, v_{e, v}^{\prime}\right\}\right), \\
& E\left(G^{\prime}\right)=\left(\bigcup_{e=(u, v) \in E_{1}}\left\{\left(v, v_{e, v}^{\prime}\right),\left(v_{e, v}^{\prime}, v_{e, u}^{\prime}\right),\left(v_{e, u}^{\prime}, u\right)\right\}\right) \cup E_{2} .
\end{aligned}
$$

Notice that $V$ has $n+2 n=3 n$ vertices and $3 n+n=4 n$ edges. It is also easy to see that the maximum degree of $V$ is 4 , and that $V$ does not have any triangle, since any triangle of $G$ contains at least one edge of $E_{1}$ (because $\left(V(G), E_{2}\right)$ is bipartite) and each edge of $E_{1}$ is split into three.

Given $G^{\prime}$ as an instance of Vertex Cover on triangle-free graphs, the reduction to the $k$-means problem is the same as before. Let $b_{i}=(0, \ldots, 1, \ldots, 0)$ be the $i$ th vector in the standard basis of $\mathbb{R}^{3 n}$. For an edge $e=\left(v_{i}, v_{j}\right) \in E\left(G^{\prime}\right)$, set $x_{e}=b_{i}+b_{j}$. The instance consists of the parameter $k=\left(\alpha_{\min }+1\right) n$ and the point set $\left\{x_{e} \mid e \in E\right\}$. Notice that the number of points is now $4 n$ and their dimension is $3 n$.

We now analyze the reduction. Note that for $k$-means, once a cluster is fixed as a set of points, the optimal center and the cost of the cluster are determined Let $\operatorname{cost}(C)$ be the cost of a cluster $C$. We abuse notation and use $C$ for the set of edges $\left\{e: x_{e} \in C\right\} \subseteq E\left(G^{\prime}\right)$ as well. For an integer $l$, define an l-star to be a set of $l$ distinct edges incident to a common vertex. The following lemma is proven by Awasthi et. al. and shows that if $C$ is cost-efficient, then two vertices are sufficient to cover many edges in $C$. Furthermore, an optimal $C$ is either a star or a triangle.

Lemma 3 ([2], Proposition 9 and Lemma 11). Let $C=\left\{x_{e_{1}}, \ldots, x_{e_{l}}\right\}$ be a cluster. Then $l-1 \leq \operatorname{cost}(C) \leq 2 l-1$, and there exist two vertices that cover at least $\lceil 2 l-1-\operatorname{cost}(C)\rceil$ edges in $C$. Furthermore, $\operatorname{cost}(C)=l-1$ if and only if $C$ is either an l-star or a triangle, and otherwise, $\operatorname{cost}(C) \geq l-1 / 2$.

\footnotetext{
${ }^{4}$ For $k=1$, the optimal solution to the $k$-means problem is the centroid of the point set. This is due to a well-known fact, see, e. g., Lemma 2.1 in [9].
} 


\subsection{Completeness}

Lemma 4. If $G$ has a vertex cover of size at most $\alpha_{\min } n$, the instance of $k$ means produced by the reduction admits a solution of cost at most $\left(3-\alpha_{\text {min }}\right) n$.

Proof. Suppose $G$ has a vertex cover $S$ with at most $\alpha_{\min } n$ vertices. For each edge $e=(u, v) \in E_{1}$, let $v^{\prime}(e)=v_{e, u}^{\prime}$ if $v \in S$, and $v^{\prime}(e)=v_{e, v}^{\prime}$ otherwise. Let $S^{\prime}:=S \cup\left(\cup_{e \in E_{1}}\left\{v^{\prime}(e)\right\}\right.$. Since $S$ is a vertex cover of $G$, for every edge $e \in E_{1}$, $S$ and $v^{\prime}(e)$ cover all three edges of $E\left(G^{\prime}\right)$ corresponding to $e$. Therefore, $S^{\prime}$ is a vertex cover of $G^{\prime}$, and since $\left|E_{1}\right|=n$, it has at most $\left(\alpha_{\min }+1\right) n$ vertices.

For the $k$-means solution, let each cluster correspond to a vertex in $S^{\prime}$, and assign each edge $e \in E\left(G^{\prime}\right)$ to the cluster corresponding to a vertex incident to $e$ (choose an arbitrary one if there are two). Each edge is assigned to a cluster since $S^{\prime}$ is a vertex cover, and each cluster is a star by construction. Since there are $4 n$ points and $k=\alpha_{m i n} n+n$, the total cost of the solution is, by Lemma 3 .

$$
\sum_{i=1}^{k} \operatorname{cost}\left(C_{i}\right)=\sum_{i}^{k}\left(\left|C_{i}\right|-1\right)=\left(\sum_{i}^{k}\left|C_{i}\right|\right)-k=\left(3-\alpha_{m i n}\right) n
$$

\subsection{Soundness}

Lemma 5. If every vertex cover of $G$ has size of at least $\alpha_{\max } n$, then any solution of the $k$-means instance produced by the reduction costs at least $(3-$ $\left.\alpha_{\min }+\frac{1}{3}\left(\alpha_{\max }-\alpha_{\min }\right)\right) n$.

Proof. Suppose every vertex cover of $G$ has at least $\alpha_{\max } n$ vertices. We claim that every vertex cover of $G^{\prime}$ also has to be large.

Claim 6. Every vertex cover of $G^{\prime}$ has at least $\left(\alpha_{\max }+1\right) n$ vertices.

Proof. Let $S^{\prime}$ be a vertex cover of $G^{\prime}$. If $S^{\prime}$ contains both $v_{e, u}^{\prime}$ and $v_{e, v}^{\prime}$ for any $e=(u, v) \in E_{1}$, then $S^{\prime} \cup\{u\} \backslash\left\{v_{e, u}^{\prime}\right\}$ is a vertex cover with the same or smaller size. Therefore, we can without loss of generality assume that for each $e=$ $(u, v) \in E_{1}, S^{\prime}$ contains exactly one vertex in $\left\{v_{e, u}^{\prime}, v_{e, v}^{\prime}\right\}$. Set $S:=S^{\prime} \cap V(G)$, thus $S$ has cardinality $\left|S^{\prime}\right|-n$. Each $e \in E_{2}$ is covered by $S$ by definition. If an $e \in E_{1}$ is not covered by $S$, at least one of the three edges of $G^{\prime}$ corresponding to $e$ is not covered by $S^{\prime}$. Thus, every edge $e \in E(G)$ is covered by $S$, so $S$ is a vertex cover of $G$. Since $|S| \geq \alpha_{\max } n,\left|S^{\prime}\right| \geq\left(\alpha_{\max }+1\right) n$.

Fix $k$ clusters $C_{1}, \ldots, C_{k}$. Without loss of generality, let $C_{1}, \ldots, C_{s}$ be clusters that correspond to a star, and $C_{s+1}, \ldots, C_{k}$ be clusters that do not correspond to a star for any $l$. For $i=1, \ldots, s$, let $v(i)$ be the vertex covering all edges in $C_{i}$, and for $i=s+1, \ldots, k$, let $v(i), v^{\prime}(i)$ be two vertices covering at least $\left\lceil 2\left|C_{i}\right|-1-\operatorname{cost}\left(C_{i}\right)\right\rceil$ edges in $C_{i}$ by Lemma 3 . Let $E^{\dagger} \subseteq E\left(G^{\prime}\right)$ be the set of edges not covered by any $v(i)$ or $v^{\prime}(i)$. The cardinality of $\left|E^{\dagger}\right|$ is at most

$$
\sum_{i=s+1}^{k}\left(\left|C_{i}\right|-\left(2\left|C_{i}\right|-1-\operatorname{cost}\left(C_{i}\right)\right)\right)=\sum_{i=s+1}^{k}\left(\operatorname{cost}\left(C_{i}\right)-\left(\left|C_{i}\right|-1\right)\right) .
$$


Adding one vertex for each edge of $E^{\dagger}$ to the set $\{v(i)\}_{1 \leq i \leq s} \cup\left\{v(i), v^{\prime}(i)\right\}_{s+1 \leq i \leq k}$ yields a vertex cover of $G^{\prime}$ of size at most

$$
s+2(k-s)+\sum_{i=s+1}^{k}\left(\operatorname{cost}\left(C_{i}\right)-\left(\left|C_{i}\right|-1\right)\right)
$$

Every vertex cover of $G^{\prime}$ has size of at least $\left(\alpha_{\max }+1\right) n=k+\left(\alpha_{\max }-\alpha_{\min }\right) n$, so we have

$$
(k-s)+\sum_{i=s+1}^{k}\left(\operatorname{cost}\left(C_{i}\right)-\left(\left|C_{i}\right|-1\right)\right) \geq\left(\alpha_{\max }-\alpha_{\min }\right) n .
$$

Now, either $k-s \geq \frac{2}{3}\left(\alpha_{\max }-\alpha_{\min }\right) n$ or $\sum_{i=s+1}^{k}\left(\operatorname{cost}\left(C_{i}\right)-\left(\left|C_{i}\right|-1\right)\right) \geq$ $\frac{1}{3}\left(\alpha_{\max }-\alpha_{\min }\right) n$. In the former case, since $\operatorname{cost}\left(C_{i}\right) \geq\left|C_{i}\right|-\frac{1}{2}$ for $i>s$ by Lemma 3 , the total cost is

$$
\sum_{i=1}^{k} \operatorname{cost}\left(C_{i}\right) \geq \sum_{i=1}^{s}\left(\left|C_{i}\right|-1\right)+\sum_{i=s+1}^{k}\left(\left|C_{i}\right|-\frac{1}{2}\right) \geq\left(\sum_{i}^{k}\left|C_{i}\right|\right)-k+\frac{\left(\alpha_{\max }-\alpha_{\min }\right) n}{3}
$$

In the latter case, the total cost can be split to obtain that $\sum_{i=1}^{k} \operatorname{cost}\left(C_{i}\right) \geq$ $\sum_{i=1}^{k}\left(\left|C_{i}\right|-1\right)+\sum_{i=s+1}^{k}\left(\operatorname{cost}\left(C_{i}\right)-\left(\left|C_{i}\right|-1\right)\right) \geq\left(\sum_{i}^{k}\left|C_{i}\right|\right)-k+\frac{1}{3}\left(\alpha_{\max }-\alpha_{\min }\right) n$.

Therefore, in any case, the total cost is at least

$$
\left(\sum_{i}^{k}\left|C_{i}\right|\right)-k+\frac{1}{3}\left(\alpha_{\max }-\alpha_{\min }\right) n=\left(3-\alpha_{\min }+\frac{1}{3}\left(\alpha_{\max }-\alpha_{\min }\right)\right) n .
$$

The above completeness and soundness analyses show that it is NP-hard to distinguish the following cases.

- There exists a solution of cost at most $\left(3-\alpha_{m i n}\right) n$.

- Every solution has cost at least $\left(3-\alpha_{\min }+\frac{\alpha_{\max }-\alpha_{\min }}{3}\right) n$.

Therefore, it is NP-hard to approximate $k$-means within a factor of

$$
\frac{\left(3-\alpha_{\min }+\frac{\alpha_{\max }-\alpha_{\min }}{3}\right) n}{\left(3-\alpha_{\min }\right) n}=1+\frac{\alpha_{\max }-\alpha_{\min }}{3\left(3-\alpha_{\min }\right)}=1+\frac{1}{3\left(10 \mu_{4, k}+28\right)} \geq 1.0013 .
$$

[1] Daniel Aloise, Amit Deshpande, Pierre Hansen, and Preyas Popat. NPhardness of Euclidean sum-of-squares clustering. Machine Learning, $75(2): 245-248,2009$.

[2] Pranjal Awasthi, Moses Charikar, Ravishankar Krishnaswamy, and Ali Kemal Sinop. The hardness of approximation of euclidean k-means. In SoCG 2015 (accepted), 2015. 
[3] Miroslav Chlebík and Janka Chlebíková. Complexity of approximating bounded variants of optimization problems. Theoretical Computer Science, 354(3):320 - 338, 2006.

[4] Sanjoy Dasgupta. The hardness of $k$-means clustering. Technical Report CS2008-0916, University of California, 2008.

[5] Irit Dinur and Samuel Safra. On the hardness of approximating minimum vertex cover. Annals of Mathematics, pages 439-485, 2005.

[6] Dan Feldman and Michael Langberg. A unified framework for approximating and clustering data. In Proceedings of the 43th ACM Symposium on the Theory of Computing (STOC), pages 569-578, 2011.

[7] Mary Inaba, Naoki Katoh, and Hiroshi Imai. Applications of weighted voronoi diagrams and randomization to variance-based k-clustering (extended abstract). In Proceedings of the 10th ACM Symposium on Computational Geometry (SoCG, pages 332-339, 1994.

[8] Kamal Jain and Vijay V. Vazirani. Approximation algorithms for metric facility location and $k$-median problems using the primal-dual schema and lagrangian relaxation. Journal of the ACM, 48(2):274 - 296, 2001.

[9] Tapas Kanungo, David M. Mount, Nathan S. Netanyahu, Christine D. Piatko, Ruth Silverman, and Angela Y. Wu. A local search approximation algorithm for $k$-means clustering. Computational Geometry, 28(2-3):89 112,2004 .

[10] Stuart P. Lloyd. Least squares quantization in PCM. Bell Laboratories Technical Memorandum, 1957. later published as [11.

[11] Stuart P. Lloyd. Least squares quantization in PCM. IEEE Transactions on Information Theory, 28(2):129 - 137, 1982.

[12] Meena Mahajan, Prajakta Nimbhorkar, and Kasturi R. Varadarajan. The Planar $k$-means Problem is NP-Hard. In Proceedings of the 3rd Workshop on Algorithms and Computation (WALCOM), pages 274-285, 2009.

[13] Michael Mitzenmacher and Eli Upfal. Probability and Computing - Randomized Algorithms and Probabilistic Analysis. Cambridge University Press, 2005. Theorem 6.3 on p. 129 in Chapter 6.

\section{Appendix A. Remark on Theorem 2}

To obtain Theorem 2, note that the proof of Theorem 17 in 3 states that it is NP-hard to distinguish whether the vertex cover has at most

$$
|V(G)| \frac{2(|V(H)|-M(H)) / k+8+2 \varepsilon}{2|V(H)| / k+12} \text { or at least }|V(G)| \frac{2(|V(H)|-M(H)) / k+9+2 \varepsilon}{2|V(H)| / k+12}
$$

vertices. By the assumption in the first sentence of the proof and because $|V(H)|=2 M(H),(|V(H)|-M(H)) / k$ and $|V(H)| / k$ can be replaced by $\mu_{4, k}$ as defined in Definition 6 in [3]. By Theorem 16 in [3], $\mu_{4, k} \leq 21.7$. 